\title{
Microscopy101
}

\section{Recipes for Consistent Selected Area Electron Diffraction Results: Part 3: Electron Diffraction Analysis Software}

\section{Scott D. Walck}

SURVICE Engineering Co., contractor to U.S. Army Combat Capabilities Development Command Army

Research Laboratory, Aberdeen Proving Ground, Aberdeen, MD 21001

scott.d.walck2.ctr@mail.mil

\begin{abstract}
Electron diffraction is an essential characterization tool for materials scientists. When using the transmission electron microscope (TEM) to perform diffraction experiments, setting up the microscope for both calibration standards and unknown materials in a consistent method ensures that dependable results are obtained. Care must also be exercised to protect digital cameras from intense transmitted and diffracted beams to avoid damage. In Parts 1 and 2 of this series, procedures were presented for recording high-quality, well-calibrated, digital SAED patterns. In Part 3, aspects of diffraction analysis software packages and the reliability of data are discussed.
\end{abstract}

Keywords: TEM, selected area electron diffraction, SAED, calibration

\section{Introduction}

In the previous articles in this series procedures for setting up the microscope and recording SAED patterns were discussed. If the procedures are followed, the resulting SAED patterns will be well-calibrated and centered with good intensities for analysis. The patterns will also be well-suited for use in electron diffraction analysis software packages. In this last installment, software packages are discussed, and results for testing the reproducibility and reliability of SAED data acquired with these procedures are given.

\section{Electron Diffraction Analysis Software}

There are several commercial and free software packages available for the analysis of electron diffraction patterns. This author regularly uses DiffTools [1,2], CSpot [3], JEMS [4], and Electron Diffraction [5] to analyze experimental spot, ring, and Kikuchi patterns. It is beyond the scope of this article to discuss all electron diffraction software packages. However, there are important features that they should include. The program should be able to simulate patterns from crystal information that is either imported or created within the software. The simulated pattern should be able to be overlaid with the diffraction pattern. The different types of diffraction patterns should be able to be indexed, for example, ring, spot, and Kikuchi patterns. It also helps to be able to interactively change the camera length to help match the pattern when the simulated pattern doesn't quite fit the experimental one. For ring patterns, it is also very useful to integrate the pattern and generate a plot that can be compared to X-ray diffraction patterns. There are three critical features that must be included in the software: pattern centering, correction of pattern distortion, and pattern calibration.
Pattern centering. To measure d-spacings and angles in diffraction patterns, the center of the pattern must be accurately positioned. The procedures outlined previously for producing a "double exposure" image provides a fiducial marker that makes pattern centering easy. DiffTools has the Locate (000) Spot command, which locates the pattern center with sub-pixel resolution by finding a geometric center of gravity for the transmitted beam. The DiffTools - Locate SADP Centre menu command can also be used to locate the pattern center. With this tool, concentric circles can be superimposed on the SAED pattern as an aid to check the results, as shown in Figure 1A. The Auto method uses a Circular Hough Transform technique to accurately find the center [6]. Figure 1B shows the manual centering of the pattern in CSpot with superimposed Au rings on the pattern. Also available in CSpot is the ability to use a circular fitting method for determining the center by positioning points on a diffraction ring (Figure 1C). The colorized zoom window helps facilitate the accurate positioning of the fitting point shown in red at the maximum intensity.

Correction for elliptical patterns. Not all microscopes are created equally. Distortions are possible with the lenses that might make the patterns non-circular, that is, elliptical. Of course, this will have an adverse effect on any measurements of d-spacings or angles. The tolerance on new microscopes is less than $1 \%$, but this needs to be checked to see if distortions are present. The good news is that with digital images, the distortion can be easily measured using a polycrystalline material that generates ring patterns, as shown throughout this article. If the rings are not circular, the pattern must be corrected, and this correction can then be applied to any diffraction pattern from an unknown material [7]. For CSpot in Figure 1C, it is seen that there are two options to find the center from the sample points, fitting either a circle or an ellipse. Dave Mitchell has also created an Ellipse Fitting Analysis routine that can measure the elliptical distortion, save the correction parameters, and apply it to all patterns collected subsequently $[8,9]$. This has the advantage of applying the distortion correction to any SAED pattern in DM immediately after it is recorded and prior to analyzing it with any software package.

Calibration. Obviously, the digital image must be calibrated in order to make measurements from it and to compare it to simulated phases. Anyone who has performed diffraction 


\section{MINITOOL Precision Micro-Tools}

1

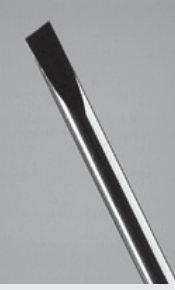

Efficient, precise and realistically proportioned instruments are ideal for microscopists. Our line of micro-tools includes needles, gravers, chisels, knives, hooks and mirrors, probes, spatulas, scribes and microrulers.

All 32 tools are offered singly or in sets of eight tools with handles. Available in tip diameters from $.025 \mathrm{~mm}$ to $1.00 \mathrm{~mm}$.

\section{www.minitoolinc.com info@minitoolinc.com}

\section{MRS-6}

We are ISO-9000 certified and ISO-17025 accredited Microscopy Calibration Standard

Now you can calibrate better from $1,000 X$ to $1,000,000 X$ !

This is our fifth generation,

traceable,

magnification

reference standard for all types (SEM, FESEM, Optical, STM, AFM, etc.) of microscopy. The MRS- 6 has multiple $X$ and $Y$ pitch patterns ranging from $80 \mathrm{~nm}( \pm 3 \mathrm{~nm})$ to $2 \mu \mathrm{m}$ and 3 bar targets from $80 \mathrm{~nm}$ to $3 \mu \mathrm{m}$. There is also a STM test pattern. Definition of the $80 \mathrm{~nm}$ pitch pattern is excellent.

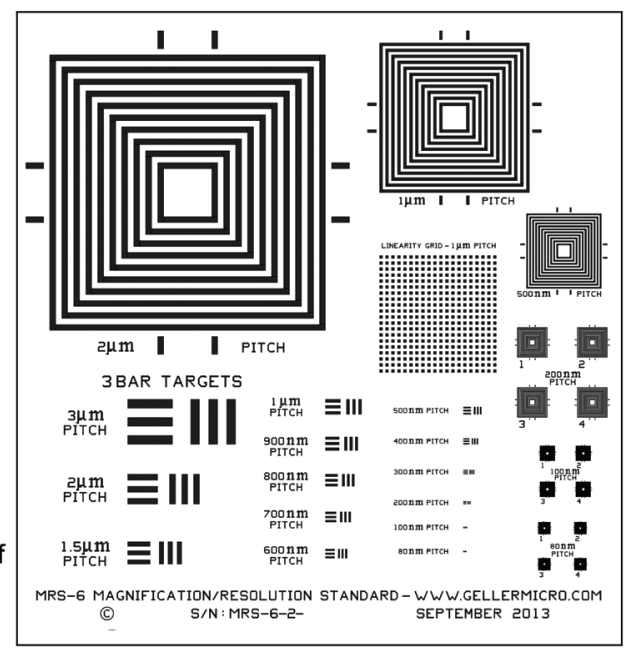

GELLER MICROÅNALYTICAL LABORATORY, Inc.

426e BOSTON ST., TOPSFIELD, MA 01983-1216 TEL: 978 887-7000 FAX: 978-887-6671 www.GellerMicro.com

\section{MEO Engineering Company, Inc.}

High Technology on a Small Scale since 2004

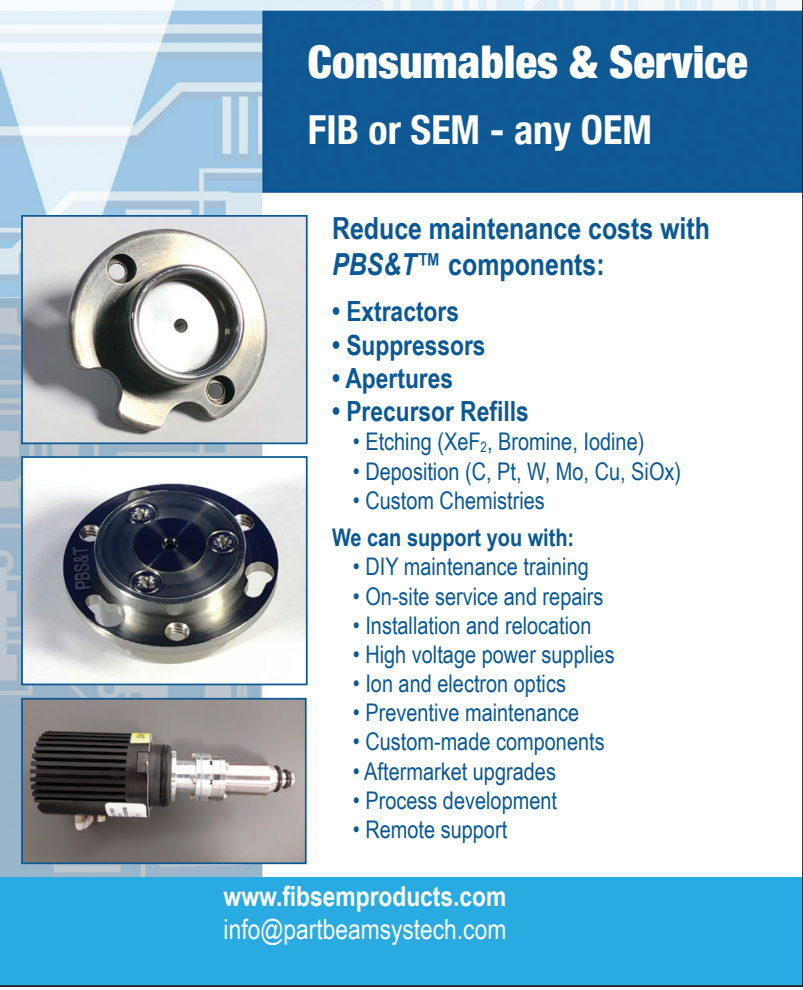

\section{MEO Engineering Company, Inc.}

High Technology on a Small Scale since 2004

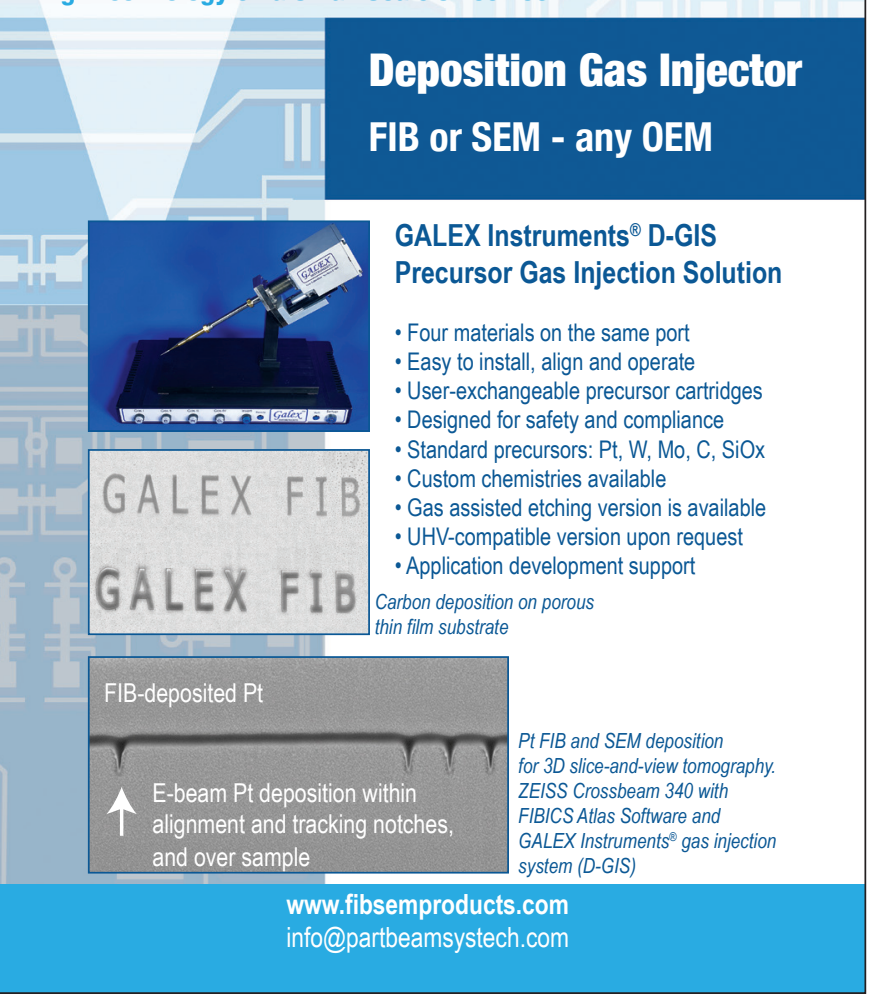



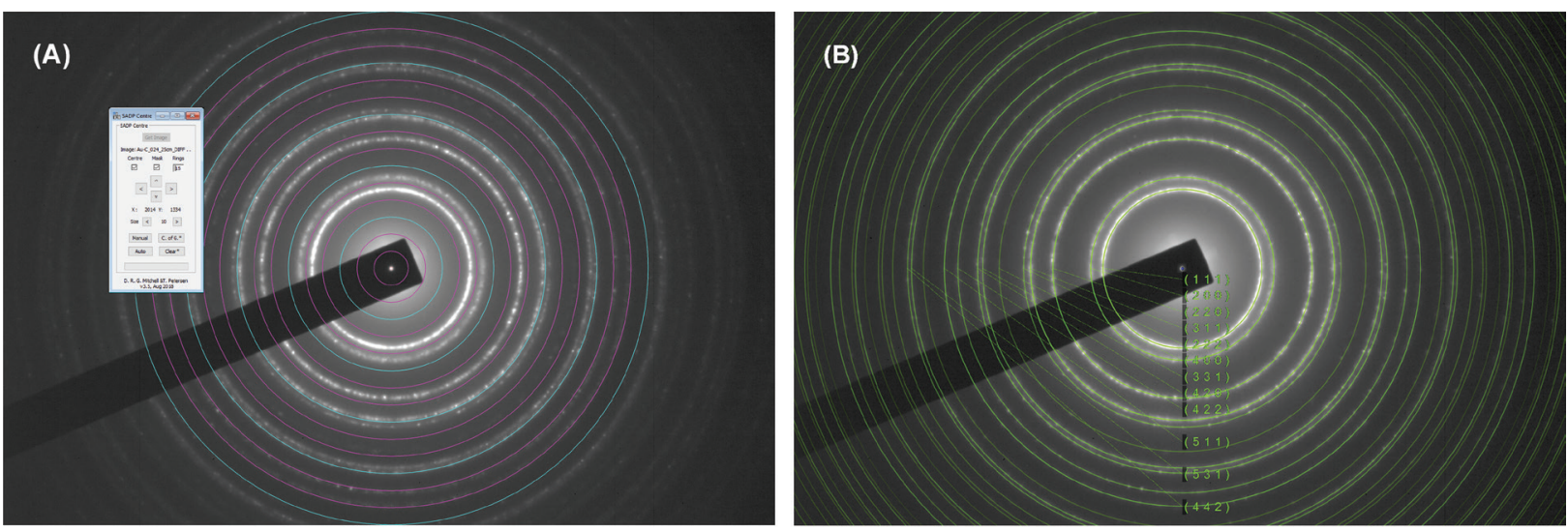

(C)
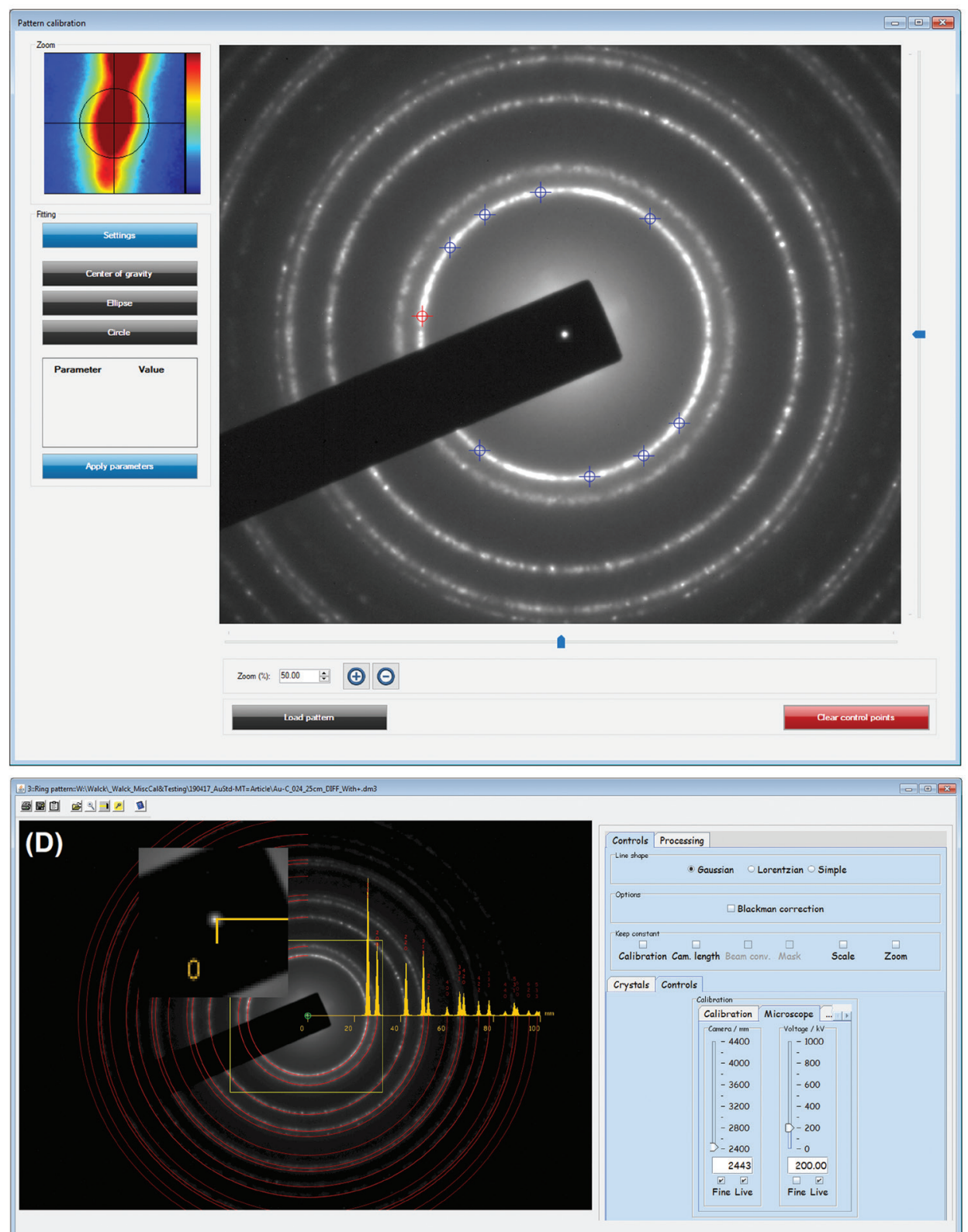

Figure 1: Centering the pattern with software. A) Centered pattern using manual adjustment in DiffTools. Three methods are available: Auto, Manual, and Center of Gravity. Concentric rings can be superimposed to help with centering the pattern. B) Manual Centering of the pattern in CSpot using the superimposed center spot Au rings. C) Pattern Centering and Corrections in CSpot. A circle or, to correct distortion, an ellipse can be fitted to the marked (111) ring to find the center of any pattern. D) Centering pattern in JEMS with superimposed rings and simulated integrated intensities. It also has a mag tool to see how well the center is set. 
analyses in the TEM has been taught the derivation for the camera constant,

$$
\lambda \mathrm{L}=\mathrm{dD}
$$

from Bragg's law, where $\lambda$ is the wavelength of the electrons, $L$ is the camera length, $\mathrm{d}$ is the $\mathrm{d}$-spacing of a reflection, and $\mathrm{D}$ is the distance on the pattern from the transmitted beam to that reflection. Students are taught that we don't know either the accelerating voltage or the camera length accurately, but we do know the product of these two parameters for a known material. To determine $\lambda \mathrm{L}$, use a known material such as gold to measure the distance on the pattern from the (000) to a known reflection with a known d-spacing and multiply the two values. For digital images, $\lambda \mathrm{L}$ can be measured in units of $\mathrm{nm}$.pixels or $\AA$.pixels. For example, if the size of the pixel elements of the camera is known, then $\lambda \mathrm{L}$ can be converted to units of $\mathrm{nm} \cdot \mathrm{cm}$. Software packages universally calculate a precise relativistic wavelength for the accelerating voltage used and then calculate the actual camera length for the nominal camera length used. An easy way that software packages calibrate the camera length is to superimpose a simulated ring pattern of the standard known material on top of an experimental ring pattern and adjust the camera length until the pattern matches, as shown in Figure 1B. DiffTools offers a gold calibration tool that works in the traditional method, where the reciprocals of the known d-spacings are plotted against the measured radius of the rings and fitted with a straight line as shown in Figure 2. From equation (1), the slope of the line is the calibration factor for the image in units of reciprocal d-spacing per pixel and is simply the reciprocal of the camera constant, $\lambda \mathrm{L}$. This approach has the benefit of giving a relative error associated with the fit, which gives an indication of the reliability for the calibration. This method will be used below to determine the reproducibility of the microscope for measuring SAED patterns using the polycrystalline gold sample. Note: for DM users, only the diameter of a single ring from a polycrystalline sample or the distance of a known reflection from a spot pattern from a single crystal pattern is used to calibrate DM (Figure 3A). Notice that DiffTools calibrates the diffraction pattern in units of $\AA^{-1}$, whereas units of $\mathrm{nm}^{-1}$ were chosen for DM. Differentiating this way allows the user to know which calibration factor was used, DiffTools or DMs. If desired, the values determined by DiffTools can be used to replace the DM calibration values so that the confidence of the calibration factor is known. To do this, take the reciprocal of the calibration, divide by the relativistic wavelength, $\lambda(\AA)$, and multiply by the camera pixel size in $\mathrm{mm}$ to get the value in camera length with units of $\mathrm{mm}$. This is the value that can be used in the edit box of Figure 3B for changing the calibration manually in DM. Notice the slight discrepancy in calibration values between DiffTools $\left(1.0320 \times 10^{-3} \AA^{-1} / \mathrm{pixel}\right)$ and DM $\left(0.010259 \mathrm{~nm}^{-1} / \mathrm{pixel}\right)$, which is a difference of $0.59 \%$. DiffTools should be better because it samples the entire intensity of the rings and more than just one ring.

\section{Can SAED Results Be Trusted?}

Lattice parameter. After all of this, it is fair to ask several questions. How reliable is the calibration factor that is used to analyze a SAED pattern? How reliable are the measurements? How reproducible is a measurement? How often does the microscope require calibration? In preparing this article, a series of SAED patterns from a polycrystalline gold calibration standard sample were collected at two nominal camera lengths, 20 and $25 \mathrm{~cm}$. Prior to each SAED pattern, the sample was moved to a random area, the " $\mathrm{Z}$ " position adjusted to the standard focus position, IL1 focused, the pattern centered on the crosshair, and then the long exposure and short exposure patterns acquired. Using calibration values that had been taken well over two years previously, DiffTools was used to calibrate the camera length, find the center of the pattern, perform a rotational

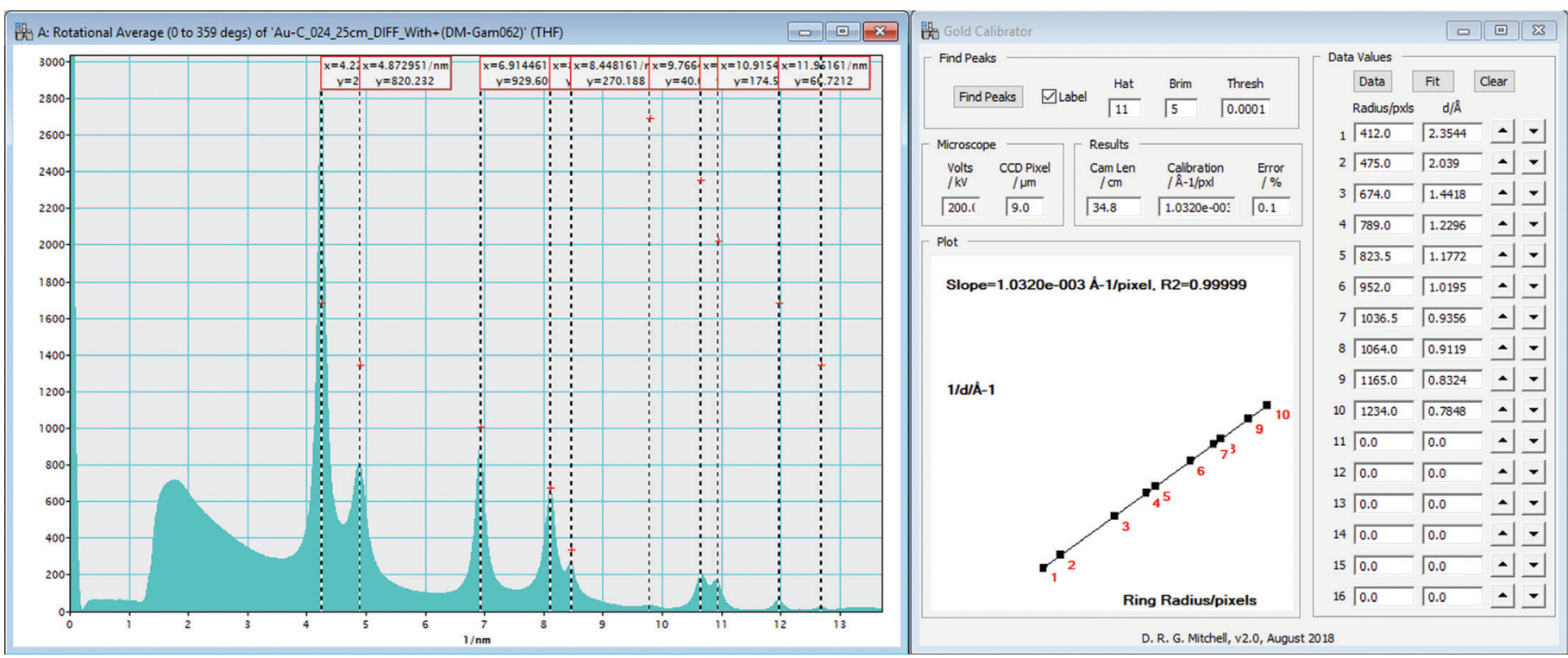

Figure 2: Screen capture of the Gold Calibration results from DiffTools. The slope of the line is the calibration factor measured in $\AA^{-1}$. pixels ${ }^{-1}$, which is the reciprocal of the camera constant. With the accelerating voltage and pixel resolution of the camera known, the camera length for the microscope is calculated. In this example, the nominal $25 \mathrm{~cm}$ camera length is actually $34.8 \mathrm{~cm}$. 
(A)

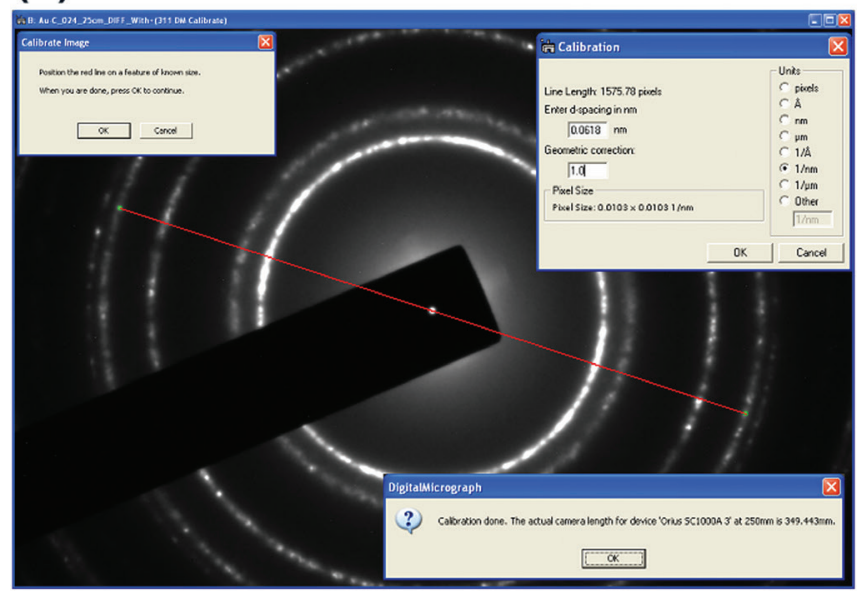

(B)

\section{(C)}
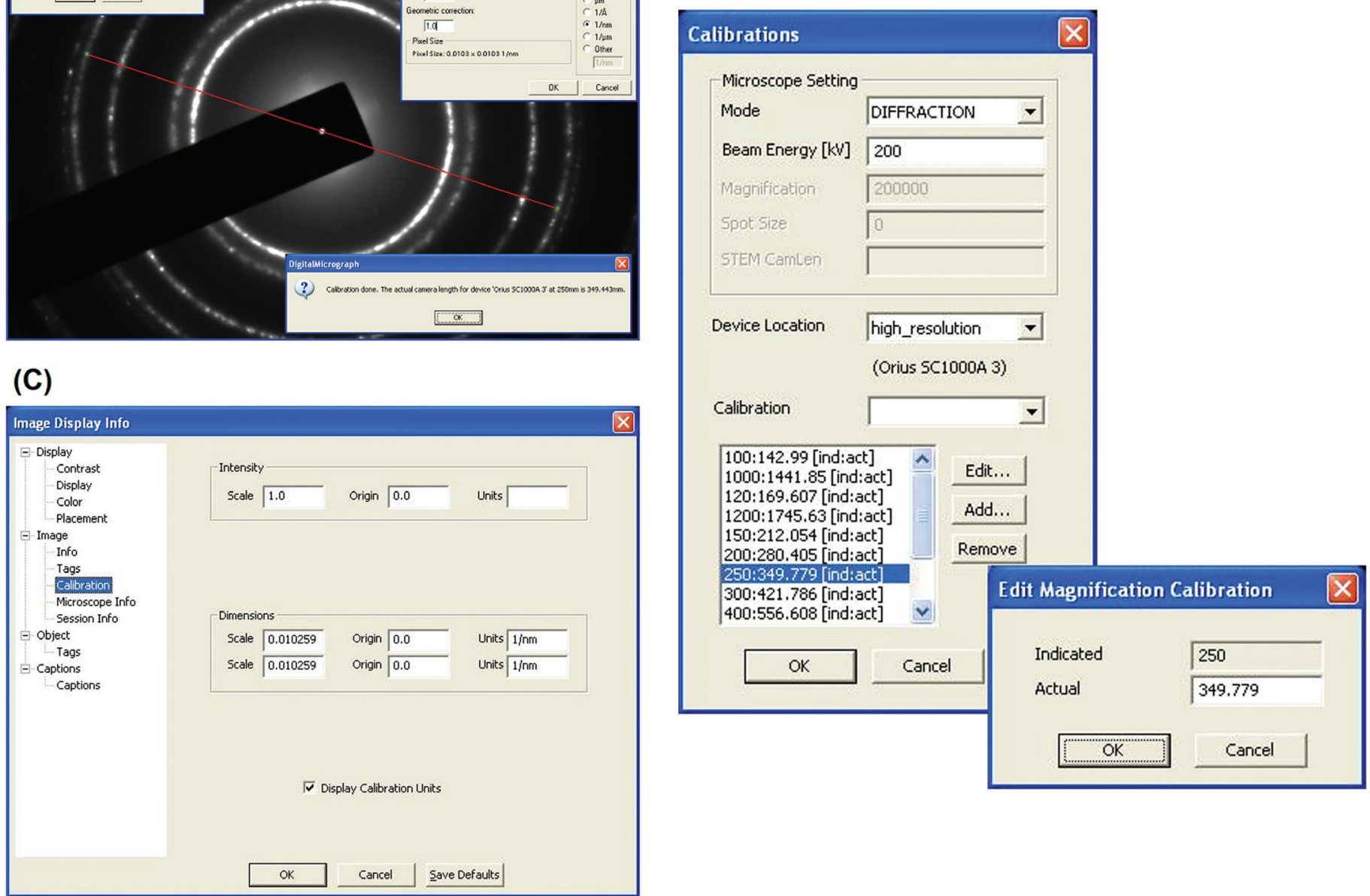

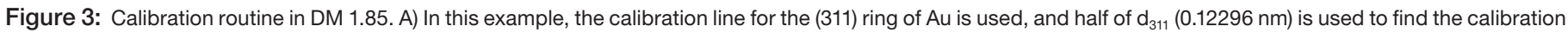

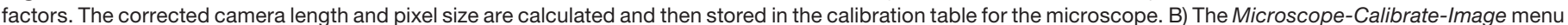
command to show the microscope calibration factors with the edit command insert are shown. C) Image Calibration information displayed for a SAED image in DM.

averaged intensity profile, and find the peaks at each position. The DiffTools-Gold Calibrator routine was then used to determine the calibration factor for the pattern at the same position using the first ten peaks of the pattern. Tables 1, 2 and 3 show the resulting $\mathrm{Au}(111) \mathrm{d}$-spacings and calibration factors for the 20 and $25 \mathrm{~cm}$ nominal camera lengths at each of the different positions (please forgive the significant digits). For each measurement of the $\mathrm{Au}$ (111) d-spacing, the percent difference from the known value is shown. For the calibration factor results, the err\% value is a measure of the linear fit. Using the first ten peaks in the patterns gave 0.99999 for the correlation coefficient value, $\mathrm{R} 2$, for every pattern measured and is therefore not shown in the tables. The tables show that the calibration factors that are over two years old still gave d-spacings that are within about $1 \%$ of the known value. In the experiment, each position required a good deal of adjustment of the " $Z$ " position prior to acquiring a pattern. Even with this, the tables show that the procedures outlined above gave very consistent results, whether measured by a d-spacing, camera length, or calibration factor. It is also interesting to note that the variation is higher for the lower camera constant. To explain this and to make it simple, let's assume that the calibration factor is known or that it has very little experimental error. To find the reciprocal d-spacing, $1 / \mathrm{d}_{\mathrm{hkl}}$ in units of $\AA^{-1}$ for a given (hkl) from a diffraction ring, the radius of the ring, $\mathrm{R}$, in pixels is multiplied by the calibration factor for the camera length, $C_{L}$, used in units of $\AA^{-1}$ pixels ${ }^{-1}$.

$$
\frac{1}{d_{h k l}}=C_{L} \cdot R
$$

The differential expression for this then becomes

$$
d\left(\frac{1}{d_{h k l}}\right)=\ln \left(\frac{1}{d_{h k l}}\right) d\left(d_{h k l}\right)=C_{L} \cdot d R
$$

Considering that the lowest $d R$ for determining the radius of the ring is one pixel, then the smallest differential for determining the d-spacing from the rotationally averaged intensity profile is given by

$$
\Delta d_{h k l}=\frac{C_{L}}{\ln \left(d_{h k l}\right)}
$$


Table 1: SAED results for $20 \mathrm{~cm}$ camera length.

\begin{tabular}{|c|c|c|c|c|c|c|c|}
\hline \multicolumn{3}{|c|}{ Au d-spacing } & \multicolumn{5}{|c|}{ DiffTools Au Calibrator Factor Results } \\
\hline Area \# & $\begin{array}{l}\text { Measured } \\
\text { Au d }_{111}(\AA)\end{array}$ & $\begin{array}{c}2.3544 \AA \\
\% \Delta\end{array}$ & $\begin{array}{c}\text { Cam Len } \\
(\mathrm{cm})\end{array}$ & $\begin{array}{c}\text { Cal }\left(\AA^{-1 / p i x}\right) \\
\times 10^{-3}\end{array}$ & err $\%$ & $\% \Delta$ From Avg & $\% \Delta$ From Old \\
\hline 201 & 2.3730 & 0.79 & 27.254 & 1.3174 & 0.10 & 0.61 & 0.82 \\
\hline 202 & 2.3656 & 0.48 & 27.299 & 1.3158 & 0.09 & 0.49 & 0.70 \\
\hline 203 & 2.3620 & 0.32 & 27.316 & 1.3152 & 0.08 & 0.44 & 0.65 \\
\hline 204 & 2.3620 & 0.32 & 27.352 & 1.3130 & 0.11 & 0.28 & 0.48 \\
\hline 205 & 2.3620 & 0.32 & 27.371 & 1.3111 & 0.11 & 0.13 & 0.34 \\
\hline 206 & 2.3584 & 0.17 & 27.384 & 1.3116 & 0.07 & 0.17 & 0.37 \\
\hline 207 & 2.3620 & 0.32 & 27.364 & 1.3115 & 0.12 & 0.16 & 0.37 \\
\hline 208 & 2.3584 & 0.17 & 27.420 & 1.3090 & 0.10 & 0.03 & 0.18 \\
\hline 209 & 2.3332 & 0.90 & 27.717 & 1.2949 & 0.10 & 1.11 & 0.90 \\
\hline 210 & 2.3296 & 1.05 & 27.751 & 1.2943 & 0.11 & 1.15 & 0.95 \\
\hline$A v g=$ & 2.3566 & & 27.423 & 1.3094 & & & \\
\hline Std Dev $=$ & 0.0139 & & 0.171 & 0.0082 & & & \\
\hline Rel Err \%= & $0.59 \%$ & & $0.62 \%$ & $0.62 \%$ & & & \\
\hline
\end{tabular}

Table 2: SAED Results for $25 \mathrm{~cm}$ camera length.

\begin{tabular}{|c|c|c|c|c|c|c|c|}
\hline \multicolumn{3}{|c|}{ Au d-spacing } & \multicolumn{5}{|c|}{ DiffTools Au Calibrator Factor Results } \\
\hline Area \# & $\begin{array}{l}\text { Measured } \\
\text { Au d }_{111}(\AA)\end{array}$ & $\begin{array}{c}2.3544 \AA \\
\% \Delta\end{array}$ & $\begin{array}{l}\text { Cam Len } \\
(\mathrm{cm})\end{array}$ & $\begin{array}{c}\text { Cal }\left(\AA^{-1} / p i x\right) \\
\times 10^{-3}\end{array}$ & err \% & $\begin{array}{c}\% \Delta \text { From } \\
\text { Avg }\end{array}$ & $\begin{array}{c}\% \Delta \text { From } \\
\text { Old }\end{array}$ \\
\hline 401 & 2.3578 & 0.14 & 34.595 & 1.0370 & 0.13 & 0.19 & 0.13 \\
\hline 402 & 2.3550 & 0.03 & 34.616 & 1.0373 & 0.09 & 0.22 & 0.15 \\
\hline 403 & 2.3578 & 0.14 & 34.605 & 1.0366 & 0.10 & 0.15 & 0.09 \\
\hline 404 & 2.3521 & 0.10 & 34.661 & 1.0357 & 0.17 & 0.06 & 0.00 \\
\hline 405 & 2.3521 & 0.10 & 34.715 & 1.0337 & 0.12 & 0.13 & 0.19 \\
\hline 406 & 2.3492 & 0.22 & 34.693 & 1.0348 & 0.09 & 0.02 & 0.09 \\
\hline 407 & 2.3521 & 0.10 & 34.691 & 1.0347 & 0.12 & 0.03 & 0.10 \\
\hline 408 & 2.3464 & 0.34 & 34.737 & 1.0339 & 0.10 & 0.11 & 0.17 \\
\hline 409 & 2.3464 & 0.34 & 34.732 & 1.0335 & 0.10 & 0.15 & 0.21 \\
\hline 410 & 2.3521 & 0.10 & 34.727 & 1.0333 & 0.08 & 0.17 & 0.23 \\
\hline$A v g=$ & 2.3521 & & 34.677 & 1.0351 & & & \\
\hline Std Dev= & 0.0040 & & 0.055 & 0.0015 & & & \\
\hline Rel Err \%= & $0.17 \%$ & & $0.16 \%$ & $0.15 \%$ & & & \\
\hline
\end{tabular}

Table 3: Calibration reproducibility $(20 \mathrm{~cm})$ check.

\begin{tabular}{|l|c|c|c|c|}
\hline Area \# & $\begin{array}{c}\text { Cam Len } \\
(\mathbf{c m})\end{array}$ & Cal $\left(\AA^{-1 / p i x)}\right.$ & err \% & R2 \\
\hline 1 & 28.221 & $1.2719 \times 10^{-3}$ & $0.11 \%$ & 0.99999 \\
\hline 2 & 27.848 & $1.2899 \times 10^{-3}$ & $0.12 \%$ & 0.99999 \\
\hline 3 & 27.897 & $1.2864 \times 10^{-3}$ & $0.09 \%$ & 0.99999 \\
\hline 4 & 27.910 & $1.2868 \times 10^{-3}$ & $0.10 \%$ & 0.99999 \\
\hline 5 & 27.990 & $1.2837 \times 10^{-3}$ & $0.10 \%$ & 0.99999 \\
\hline 6 & 27.875 & $1.2881 \times 10^{-3}$ & $0.09 \%$ & 0.99999 \\
\hline 7 & 27.856 & $1.2892 \times 10^{-3}$ & $0.10 \%$ & 0.99999 \\
\hline 8 & 28.046 & $1.2805 \times 10^{-3}$ & $0.10 \%$ & 0.99999 \\
\hline 9 & 27.918 & $1.2868 \times 10^{-3}$ & $0.10 \%$ & 0.99999 \\
\hline 10 & 27.242 & $1.3186 \times 10^{-3}$ & $0.12 \%$ & 0.99999 \\
\hline Avg= & 27.880 & $1.2882 \times 10^{-3}$ & & \\
\hline Std Dev $=$ & 0.251 & $1.19 \times 10^{-5}$ & & \\
\hline Rel Err \%= & $0.90 \%$ & $0.93 \%$ & & \\
\hline
\end{tabular}

For the 20 and $25 \mathrm{~cm}$ camera lengths, $\Delta \mathrm{d}_{111}$ are 0.0015 and 0.0012 , respectively. Since the calibration factor, $C_{L}$, will decrease as the camera length increases, the error associated with positioning of the peak will improve for a given (hkl), as the tables show. Equation 4 indicates two things should be done experimentally to improve the measurement of a sample's lattice parameters: the highest camera length and the largest reciprocal lattice vector, that is, largest possible index in the pattern, should be used. However, care must be exercised in using too high a camera length because then fewer peaks are available to determine the calibration factor in the linear fitting routine, resulting in a higher uncertainty.

Error in " $Z$ " height positioning. Part 1 of this series of articles emphasized the importance of having the sample at the correct height with the microscope at standard focus and IL1 grabbing the back focal plane. How critical are these parameters? To investigate, a series of ten SAED patterns at different " $Z$ " height positions were acquired, and the $\mathrm{Au}_{111} \mathrm{~d}$-spacings and 


\section{Incorrect "Z" Position SAED Results}

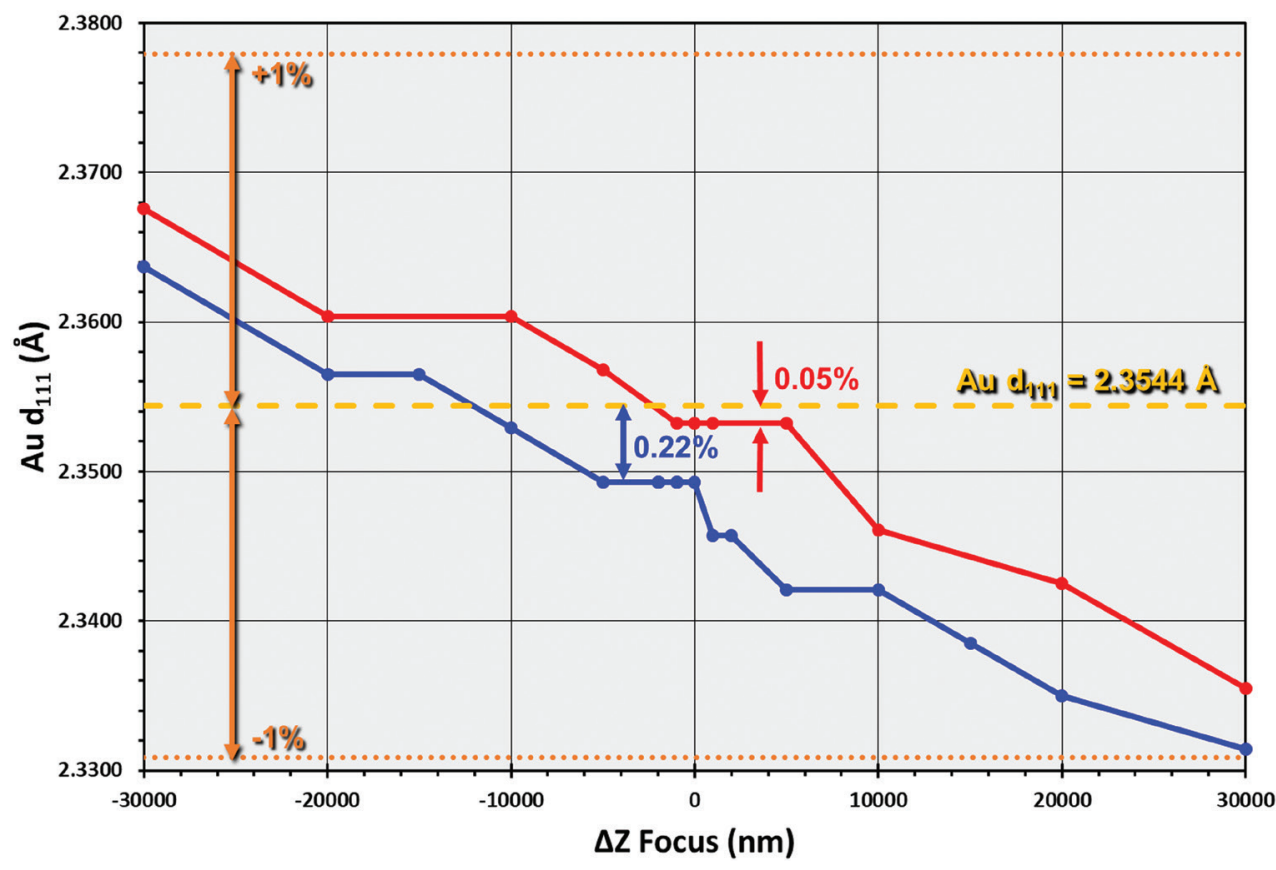

Figure 4: Variation of $\mathrm{Au}(111) \mathrm{d}$-spacing value with the sample positioned at the incorrect height in the microscope. Red and blue curves acquired on different days.

\section{Incorrect "Z" Position Calibration Factor}

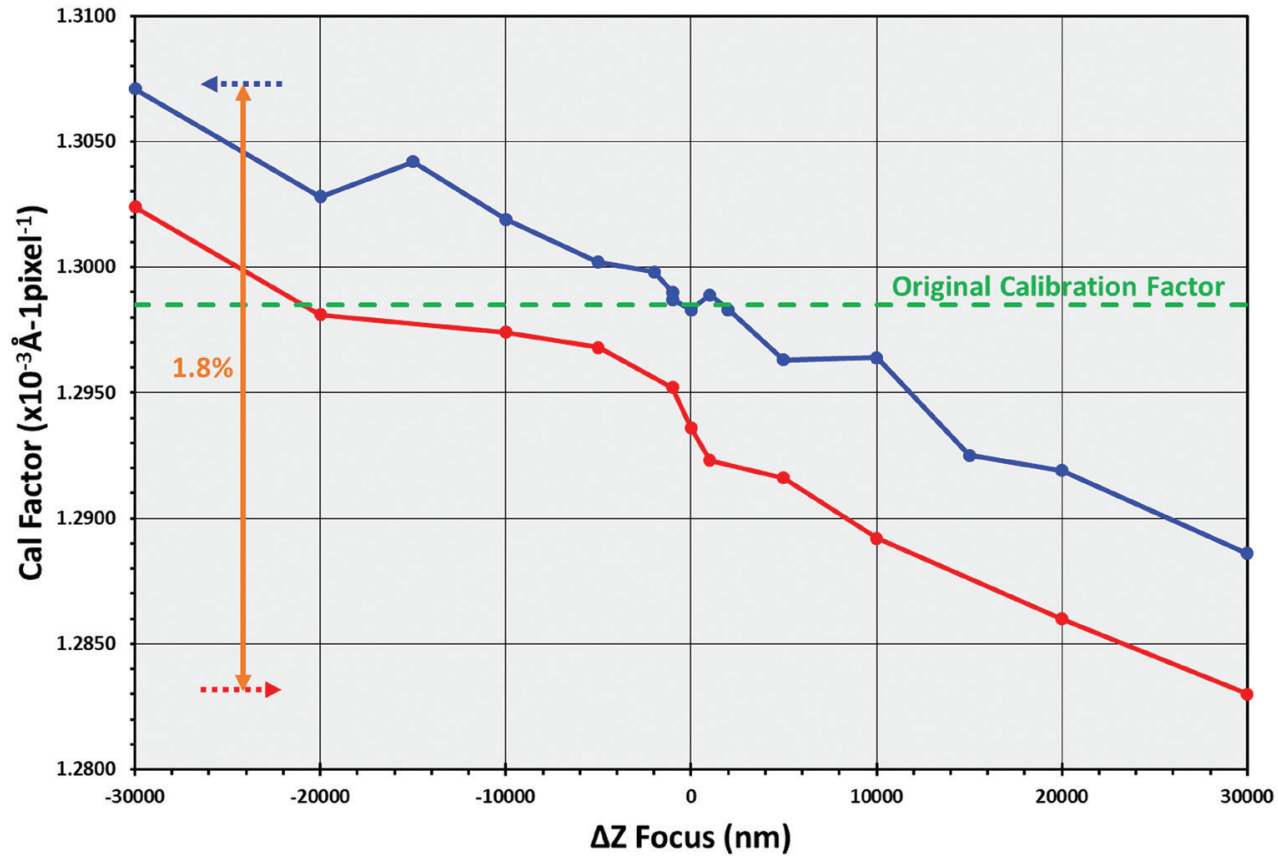

Figure 5: Variation of calibration factor with sample positioned at the incorrect height in the microscope. Red and blue curves acquired on different days.

resulting calibration factors were measured. Ten random positions were first saved at the height of the original entry of the TEM holder so that the " $Z$ " position would have to be adjusted for each pattern. The first SAED pattern was acquired at the standard position as explained above and designated the $0 \mathrm{~nm}$ focus.
Subsequent patterns were acquired by first defocusing the objective lens by a specific amount and then changing the " $Z$ " position until a minimum contrast condition was achieved. Then IL1 was adjusted so that the (000) spot was minimized. DiffTools was used to set the calibration of the pattern from the stored value, locate the center of the pattern, do a rotational average, and find the peaks as described above. The measured $\mathrm{Au}_{111} \mathrm{~d}$-spacing was noted and then the DiffTools-Gold Calibrator routine run to find the calibration factor.

The results are shown in red in Figures 4 and 5, respectively. Two results were so surprising that the experiment was repeated a few days later, shown in blue. First, note that the difference from the true value for the $\mathrm{Au}(111) \mathrm{d}$-spacing for the worse case was only $0.22 \%$. That is not so surprising considering the results from the tables. However, what is surprising is that the $\mathrm{d}$-spacing values are within $1 \%$ from the true value, even when the " $Z$ " position of the sample is a rather distant $30 \mu \mathrm{m}$ away from where it should be. And, with a difference of $60 \mu \mathrm{m}$ of positioning error, the calibration factors are within 1.8\% of each other. However, what was really surprising and what caused the suspicion of something being wrong with the experiment were the four values of $d$-spacings within $5 \mu \mathrm{m}$ of the zero-focus position. All four values had the exact same values to four decimal digits, even after double-checking the calculations. Repeating the experiment gave a similar result with four values agreeing within four decimal digits, although offset from the original data, as shown in the blue curve in Figure 4. Several factors may be responsible. If Equation 4 is considered for a camera length of $20 \mathrm{~cm}$, the (111) ring has too low of a ring diameter in pixels for the change in diameter to have much effect. The difference in the diameter of the ring when the sample height is changed from the zero position is probably also compensated somewhat by the refocusing of the IL1 lens. Using the (311) ring (fourth ring) gave similar results, but some differences were seen in the values when the (420) ring (eighth ring) was used. 


\section{"Take-Aways"}

There are a number of things that the reader can take away from this series of articles.

1) If the microscope is consistently set up in the same way, diffraction results can be very reliable and accurate.

2) Acquiring SAED patterns with a "double exposure" transmission beam included makes centering the pattern in diffraction analytical software much easier.

3) The lattice parameters will be a bit more accurate when a larger diameter ring (or spot) is used to calculate them. Use as large a camera length as possible, but remember that the number of rings in the standard is fewer, and so the error in the calibration factor will increase.

4) The big surprise here is how careless an operator can be and still obtain reasonable values for $\mathrm{d}$-spacings. Even very large departures from the correct height position gave d-spacings that were within $1 \%$ of the true value. However, by being very careful, it was possible to obtain values that were well within $0.5 \%$. This author usually tells users that if they get a value within $1 \%$, be happy with it. If it is over about $1.5 \%$, then something isn't right. Perhaps a re-evaluation of that advice is warranted.

\section{Acknowledgements}

The research reported in this document was performed in connection with contract/instrument W911QX-16-D-0014 with the U.S. Army Research Laboratory. (As of January 31, 2019, the organization is now part of the U.S. Army Combat Capabilities Development Command [formerly RDECOM] and is now called CCDC Army Research Laboratory.)

The views and conclusions contained in this document are those of the author and should not be interpreted as presenting the official policies or position, either expressed or implied, of the CCDC Army Research Laboratory or the U.S. Government unless so designated by other authorized documents. Citation of manufacturer's or trade names does not constitute an official endorsement or approval of the use thereof. The U.S. Government is authorized to reproduce and distribute reprints for Government purposes notwithstanding any copyright notation hereon.

\section{References}

[1] DRG Mitchell, Microsc ResTech 71 (2008) 588-93.

[2] DRG Mitchell, DiffTools, Dave Mitchell's DigitalMicrograph $^{\text {TM }}$ Scripting Website, www.dmscripting.com.

[3] K Sztwiertnia and K Kudłacz, CSPOT, software available from CrystOrient, www.crystorient.com.

[4] P Stadelmann, JEMS, software available from JEMSSAAS, www.jems-saas.ch.

[5] J-P Morniroli, Electron Diffraction, software available from www.electron-diffraction.fr.

[6] DRG Mitchell, Ultramicroscopy 108 (2008) 367-74.

[7] SD Walck and P Ruzakowski-Athey, Microsc Microanal 4 (Supp 2) (1998) 342-43.

[8] DRG Mitchell and JA Van den Berg, Ultramicroscopy 160 (2016) 140-45.

[9] DRG Mitchell, EFAnalysis.gtk, Dave Mitchell's DigitalMicrograph $^{\text {TM }}$ Scripting Website, www.dmscripting.com.

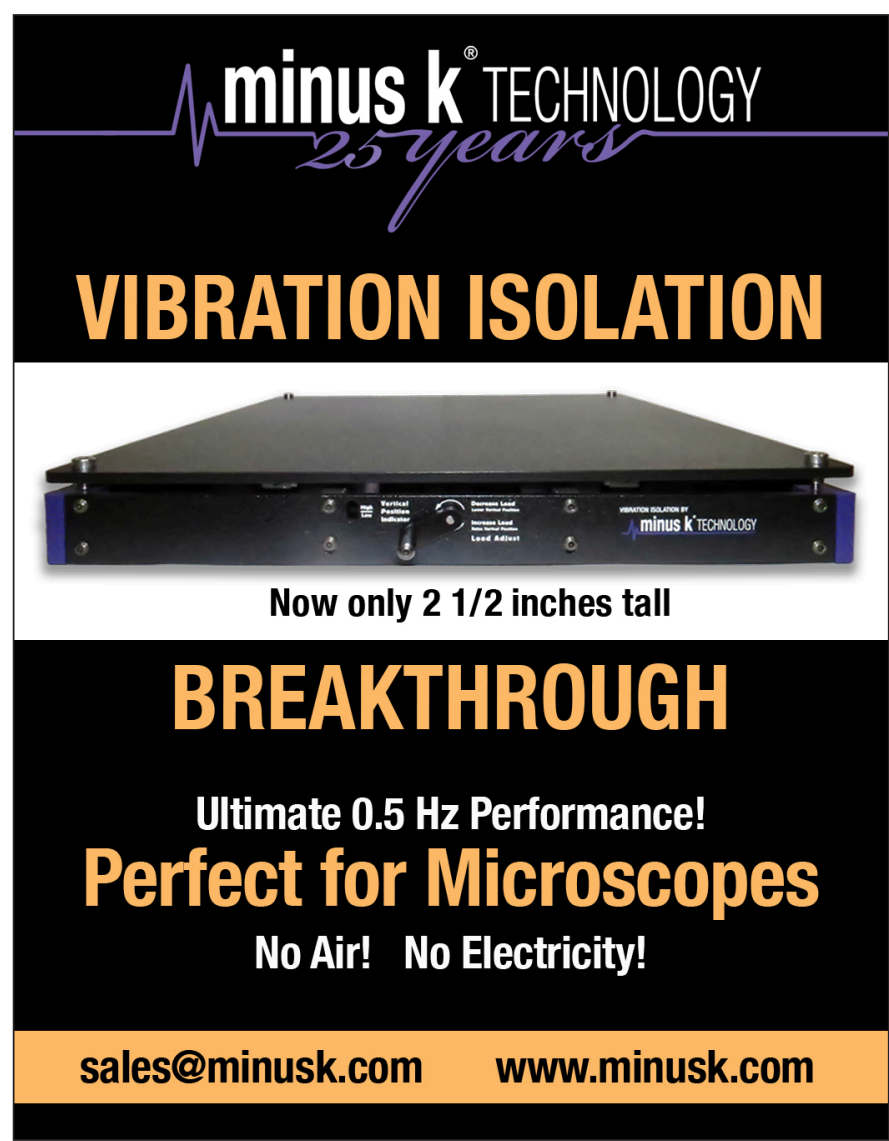

\section{SEM Scintillators \& Light Guides} Light Guide Recoating Services too!

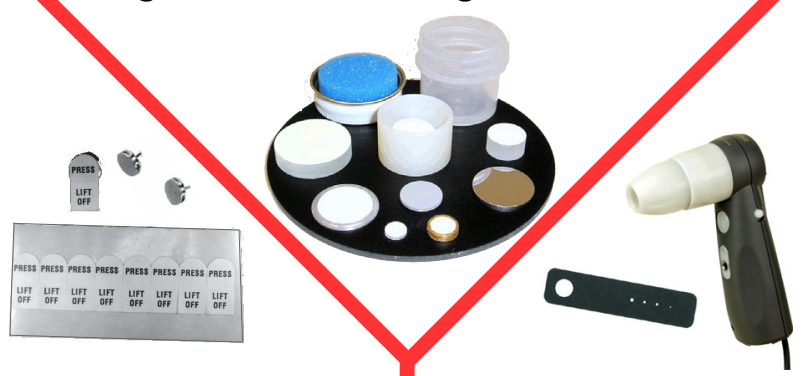

Conductive Adhesives, Apertures, ProScopes ${ }^{\mathrm{TM}}$ Coatings \& Tabs! Vacuum Supplies \& More!

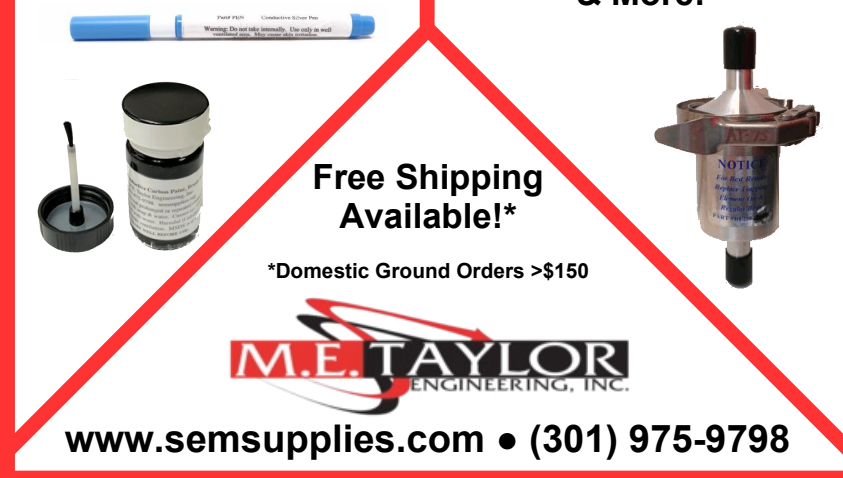

